Originalveröffentlichung in: Bader, Lena ; Gaier, Martin ; Wolf, Falk (Hrsgg.):

Vergleichendes Sehen, München 2010, S. 19-42 (Eikones)

\title{
Bricolage mit Bildern. \\ Motive und Motivationen vergleichenden Sehens
}

Lena Bader

\section{Impulse}

Vom Paragone über die Kunstkammer und die Querelle des anciens et modernes bis hin zu kennerschaftlichen Kunstbetrachtungen im 18. und 19. Jahrhundert, durchzieht das vergleichende Sehen die Vorgeschichte der akademischen Kunstgeschichte. Im Rahmen stilhistorischer Vergleiche und unterstützt durch die neuen Medien Fotografie und Lichtbildprojektion erfährt das Prinzip spätestens in der zweiten Hälfte des 19. Jahrhunderts seine methodologische Durchdringung als kunsthistorische Sehtechnik. Auch jenseits der Kunstgeschichte ist vergleichendes Sehen gleichermaßen feststehende Formel und topischer Bezugspunkt (populär)wissenschaftlicher und künstlerischer Praktiken. Wie aufschlussreiche Dokumente einer noch zu rekonstruierenden Bildgeschichte vergleichenden Sehens zeigen, wird das Verfahren hier wie dort als markantes Moment zur Auszeichnung besonderer Bilderfahrungen inszeniert [Abb. 1].

Als `Königsweg und `Zauberstab < eilt dem Verfahren jedoch ein ambivalenter Ruhm voraus. "Suspicions about comparitism «, die im Rahmen vergleichender Literaturwissenschaft intensiv reflektiert wurden, scheinen in diesem Zusammenhang aktualisiert. ${ }^{1}$ Unterstützt durch den sschlechte[n] Ruf der Kategorie des 


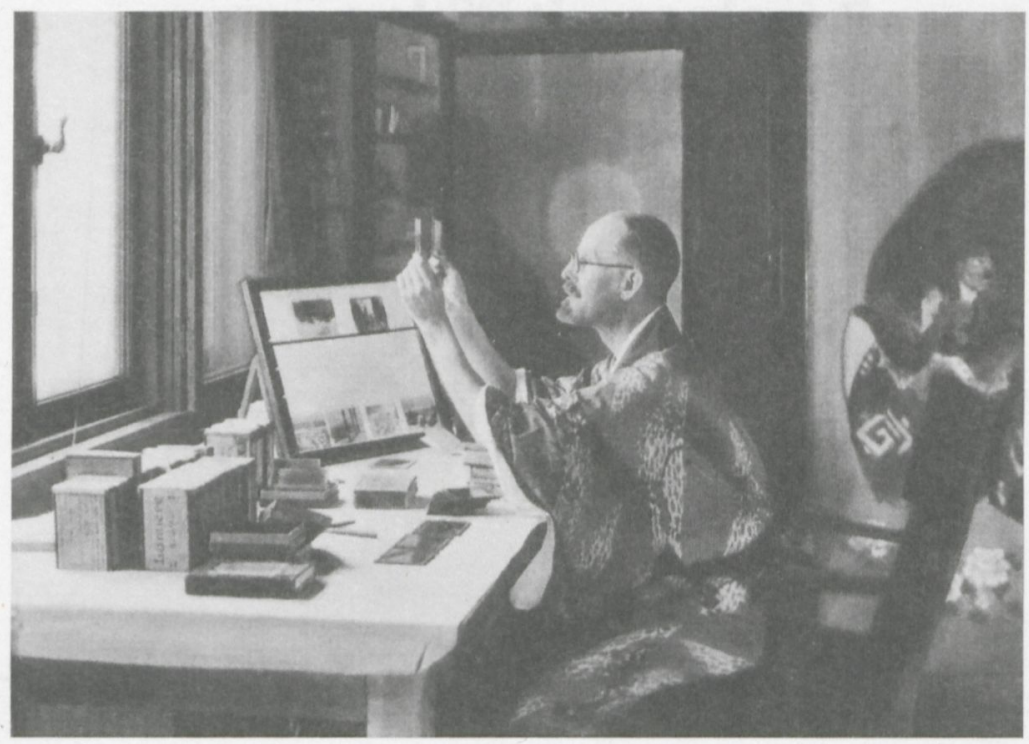

1 Burton Holmes, Reisender, Fotograf und Dokumentarfilmer, um 1930.
Vergleichs $",{ }^{2}$ der insbesondere von Seiten der Kulturwissenschaften aber auch im Rahmen neuerer Diskussionen über >Transfer^ und ,Vergleich markiert wurde, scheint sich die kunsthistorische Kritik am »auktorialen Gestus kunstgeschichtlicher Darstellung « ${ }^{3}$ in jüngster Zeit umso vehementer gegen die komparatistische Methode gerichtet zu haben. Für eine kritische Auseinandersetzung mit Möglichkeiten und Grenzen des Verfahrens hat sich insbesondere Peter Geimer ausgesprochen, um die Frage aufzuwerfen, inwiefern vergleichendes Sehen die Beschäftigung mit Bildern auf den Nachweis von »Kontinuitäten im Bildgebrauch " einengt. ${ }^{4}$

Im Zuge der Auseinandersetzung mit den medialen Grundlagen des Fachs, die auch die Technik der Dia-Doppelprojektion in den Fokus rückte, haben sich die kritischen Anmerkungen abermals verschärft. André Malraux’ Diktum, die Kunstgeschichte sei, seitdem sie »in den letzten hundert Jahren den Händen der Fachleute immer mehr entgleitet, [...] eine Geschichte des Photographierbaren geworden $«,{ }^{5}$ wurde auf eine Kunstgeschichte des Vergleichbaren zugespitzt, um die Dia-Doppelprojektion als strenges Dispositivoder wie Robert S. Nelson vorschlägt: als »tekmerion«-kritisch zu befragen:

»[...] if slides are accepted as paintings, the normal state of affairs, then arguments based upon slides alone are persuasive, even if the evidence only exists within the rhetorical/ technological parameters of the lecture itself. Such is the 

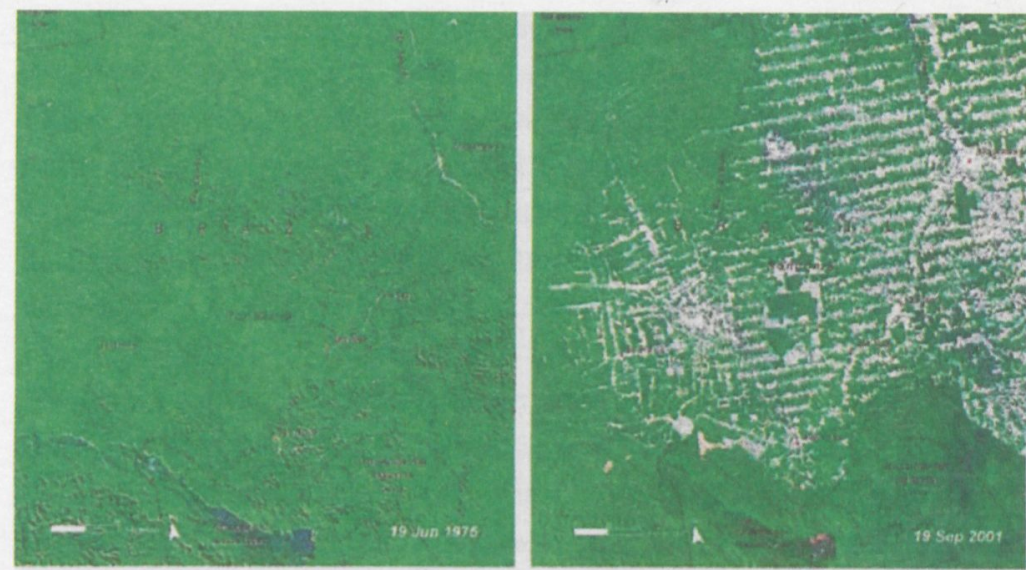

Amazonas-Fischgräten: In diesem Gebiet des brasiliamischen Bundesstaats Rondonia war ro75 noch alles grün. Die Bundesstraße BR-364 awiscben Cuiaba und Porto Velbo ließ seitber arbeits- und landsuchende Menseben in Massen in das Gebiet - und Holz in Massen benuts. Die sids charakteristisch von einer Hauptverkebrsader fischgrätenartig ausbreitenden Rodungen sind im Bild redbts cbenso zu erkennen wie der graue Ton der gerodeten Flädben, auf denen aufgrund des Näbrstoffimangels des Bodens und der Erosion keine Landwirtschaft möglicb ist.

case, for example, when objects of greatly different sizes and from unrelated cultures are regarded as comparable because they appear side by side in the slide lecture. ${ }^{6}$

2 Illustration zum Artikel nZwei Bilder sagen mehr als tausend Worte", F.A.Z., 13.06.2005.

Mit Blick auf die »seit dem 19. Jahrhundert in kunsthistorischen Übungen eingesetzten Verfahren des vergleichenden Sehens mithilfe der Dia-Doppelprojektion, die in visueller Beweisführung Stilvergleiche ermöglichte und Evidenzen provozierte«, wurde auch von kulturwissenschaftlicher Seite das Prinzip als eine »visuelle Überrumpelungskommunikation « der Kunstgeschichte kritisch zur Diskussion gestellt. ${ }^{7}$ Die verengende Konturierung, die darin zum Ausdruck kommt, konnte durch die Verbreitung populärwissenschaftlicher Formen anschaulicher Gegenüberstellungen bestärkt werden, wenn Bildpaare extremer Polarisierung (Original/Fälschung, richtig/falsch, vorher/nachher) die visuelle Komparatistik vorrangig in den Dienst illustrierender Bildnachweise oder strenger Kausalzusammenhänge stellen [Abb.2].

Auch Kategorisierungsversuche wurden vorgeschlagen, um beispielsweise Bildvergleich und Einzelbildwahrnehmung zu differenzieren:

»Wenn diese [d.h., die Einzelbildwahrnehmung] einen affektiv-personalisierten, bisweilen im engeren Sinne erotischen Charakter hat, so ist das Vergleichen jedweder Gegenstände, auch der visuellen, im Kern eine intellektuelle 
Operation. Die Wahrnehmung von Bildzusammenstellungen setzt beim Rezipienten die Fähigkeit zur Kategorienbildung voraus. Sie besteht darin, das Gemeinsame und das jeweils Eigene der zu einem binären hyperimage zusammengestellten Bilder-sei es auf inhaltlich-ikonographischer, sei es auf formal-stilistischer Ebene-begrifflich $\mathrm{zu}$ fassen. $\iota^{8}$

$\mathrm{Ob}$ und inwiefern zwischen Versenkung und Vergleich geschieden werden sollte, wäre näher zu bestimmen und mit Blick auf die Rolle der Imagination zu präzisieren. Auch scheint es geboten, das Zusammenspiel von Bild und Text als eine "Sonderform der 'Bildkritik« zu befragen, wenn beispielsweise »in der Ekphrase visuelle Korrespondenzen und Konkurrenzen " erzeugt werden, die Bilder auf unterschiedlichen Ebenen miteinander kommunizieren lassen. ${ }^{9} \mathrm{Zu}$ Recht wurde zudem pointiert, dass Vergleichbarkeit jeweils unterschiedlich motiviert sein kann, so dass "formale Ähnlichkeit nur ein Kriterium neben vielen anderen ist «. ${ }^{10}$

Vor dem Hintergrund neuerer bildtheoretischer Ansätze erscheint die Engführung des vergleichenden Sehens als »Beleg oder Argument $\aleph^{11}$ zumindest dann problematisch, wenn sie sich der Unergründlichkeit des Visuellen verschließt: „Wie könnte das, was wir den Bereich des Visuellen nennen, im strengen Wortsinn nachweisbar sein, im Sinne von swissenschaftlich`, da es selber kein Wissensgegenstand oder Wissensakt, kein Thema oder Begriff ist, sondern lediglich Wirkung auf die Blicke? «12 Wie Georges DidiHuberman nahe legt, wäre es angemessen, (die Reflexion über) das vergleichende Sehen aus dem Bann des Sichtbaren zu befreien:

"Zwei Perspektiven, die unter dem Blick einer dritten zusammentreffen. Eine Montage herzustellen, meint nicht, Dinge einander anzugleichen, sondern Ähnlichkeiten aufblitzen zu lassen und jede Gleichsetzung unmöglich zu machen. Ähnlich (semblable) zu sein, meint weder scheinbar (semblant) zu sein noch identisch zu sein. « $^{13}$

Die widersprüchlichen Bestimmungen sind bezeichnend. Sie resultieren aus der Diskrepanz zwischen stärker medientechnisch orientierten und bildtheoretischen Perspektiven, und deuten zugleich auf eine nachhaltige Ausblendung bildkritischer Fragen von Seiten der Wissenschaftsgeschichte der Kunstgeschichte. ${ }^{14}$ Ungeachtet der damit einher gehenden Wertungen markieren die 
apodiktischen Beobachtungen indes-teils implizit, teils explizitdie Wirkungsmächtigkeit anschaulicher Gegenüberstellungen. Es wäre lohnenswert, verschiedene Manifestationen vergleichenden Sehens in Wort und Bild aus dieser Perspektive näher zu befragen - von populären und (natur)wissenschaftlichen Formen vergleichenden Sehens über künstlerische Auseinandersetzungen mit dem Thema und dessen Bestimmung als kunsthistorische Sehtechnik bis hin zur Affinität bildtheoretischer Ansätze zu polaren Grundbegriffen. Die im Folgenden skizzierten Beobachtungen aus dem Kontext der Kunstgeschichte um 1900 zeigen vor diesem Hintergrund einen vergleichsweise kleinen, aber bedeutenden Ausschnitt einer komplexen Bild- und Problemgeschichte des vergleichenden Sehens.

\section{Visuelle Demonstration}

In seiner Schrift Vergleichendes Sehen im Dienste der Augen-, Geistes- und Geschmacksbildung nennt Max Brethfeld drei "Grundformen« zur Charakterisierung der vergleichenden Methode:

»a) Schlecht und gut-gut und besser, gut und auchgut.

b) Einzelformen in Gegensätzen-Reihen in Entwicklungsstufen oder in Wertreihen der Gestaltung. c) GegensatzVergrößerung-Vereinzelung. Gegensatz: wenn ich Hans Thoma und Käthe Kollwitz gegenüberstelle. Vergrößerung: wenn ich Erscheinungen der Kinderhandschrift an den weißen Wandschirm projiziere. Vereinzelung: wenn ich den Ausdruck des Auges in einem lenbachschen Bismarckbilde oder die Augen der Maria aus Gebhardts Auferweckung des Lazarus zeige. ${ }^{15}$

Gezielt befragt Brethfeld das vergleichende Sehen als »Bildungsmittel der Schule«. Seine 1931 veröffentlichte Untersuchung steht im Kontext der Schulreform und huldigt neben Kunstwart und Dürerbund namentlich Ferdinand Avenarius und insbesondere Paul Schultze-Naumburg als Pioniere und Wegbereiter des vergleichenden Sehens. ${ }^{16}$ Das Verfahren sei dabei vorrangig »in der Form von Beispiel und Gegenbeispiel« appliziert worden, wogegen Brethfeld durch Erörterung der »drei Kunstmittel des vergleichenden Sehens« zeigen möchte, dass die Methode »über ihren ursprünglichen Rahmen hinaus nach verschiedenen Seiten hin erweitert und verfeinert werden " kann. ${ }^{17}$ Emphatisch unterstreicht er in der Folge von Johann H. Pestalozzis reformpädagogischen Bestrebungen die Bedeutung des Schaumaterials: Neben "Schauschränke[n] und 
3 Giovanni Morelli, Kunstkritische Studien über italienische Malerei, 1893.
Dio Galerie nu Berlin.

leichter merken kônnen. Wïhrend Turn knollig und eckig inh, zieht Costo seine Formen stets in die Länge. $\mathrm{Zu}$ gaterletat mass ich noch bemerken, dass die landuchaftlichon Hintergründe von Costa, die sewöholieh die Ausicht aufs recen Feinger tiseher Auffassung ihresgleichen unter des Landachaften

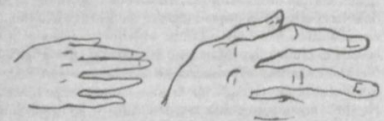

Head dee Lorvans Conts.

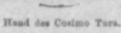

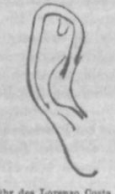

der Zeitgenossen suchen. Ich verweise namentlich auf diejenige, welebe in der merkwürdigen Darstellung des Hofes der Elisabetta Gonzaga in der Louvregalerio vorkommt (Braun 86)

Ein trockener und beschrinkter Ferrareser Maler, der aber noch im 16. Jahrbundert die Reinheit des 15 .

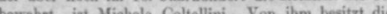
Berliner Galerie zwei, ja wol auch drei Bilder, denn
Schaukästen" sei insbesondere der Projektionsapparat "eines der wirksamsten Hilfsmittel im Dienste des vergleichenden Sehens «. ${ }^{18}$ Ohne auf die frühen Bestrebungen im Rahmen der akademischen Kunstgeschichte zu verweisen, fügt Brethfeld bedauernd hinzu, er habe "noch niemals erlebt, daß der Apparat in der Schule auch planmäßig in den Dienst des vergleichenden Sehens gestellt worden wäre". Sein Plädoyer ist eindeutig: Es gelte, das vergleichende Sehen als »ein Bildungsmittel allerersten Ranges « anzuerkennen. Seine Forderung: die »Einschaltung von planmäßigen Übungen im vergleichenden Sehen ${ }^{19}{ }^{19}$

Die Ausblendung der Kunstgeschichte ist symptomatisch. Programmatische Abgrenzungen wurden von beiden Seiten markiert. Das gilt auch für vergleichbare Mitteilungen von Seiten namhafter Kunsthistoriker - wenn beispielsweise Karl Woermann daran erinnert, die Formel vom »vergleichenden Bilderstudium " "nach Maßgabe des vergleichenden Sprachstudiums « geprägt zu haben und das Verfahren mit der Arbeit Giovanni Morellis in Verbindung bringt, d. h. in erster Linie mit Fragen der Zuschreibung [Abb. 3]. Das vergleichende Sehen steht hier für "eine neue Methode des Bilderstudiums«, wobei der Fokus explizit auf der »Beglaubigung durch scharfe Beobachtung naturwissenschaftlich greifbarer Einzelheiten " liegt. ${ }^{20}$ Während Brethfeld den Akzent auf die visuelle Demonstration legt, unterstreicht Woermann das Prinzip der visuellen Examination. In diesem Sinne hatte er 1887, im Vorwort zum 


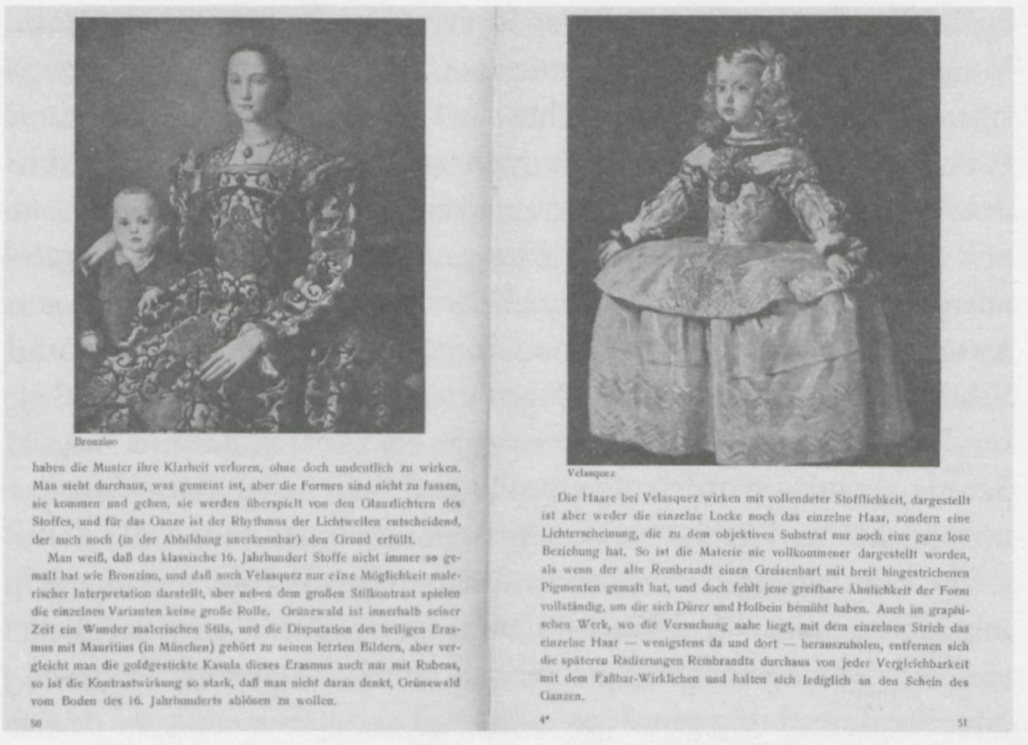

Katalog der Dresdener Gemälde-Galerie, das vergleichende Sehen gegen den »Verdacht willkürlicher Umtaufen« angeführt:

"Das vergleichende Bilderstudium ist eine junge Wissenschaft. [...] Die allgemein anerkannten Ergebnisse des vergleichenden Bilderstudiums mehren sich von Jahr zu Jahr. Es steht zu hoffen, dass in nicht zu ferner Zeit Meinungsverschiedenheiten über die Urheber aller bedeutenderen Bilder der öffentlichen Galerie kaum noch möglich sein werden. $\ll^{21}$

Die Methode, von untergeordneten Details auf die Handschrift eines Künstlers zu schließen-auch Heinrich Alfred Schmid nennt sie im Sinne Woermanns eine rationelle Methode $^{22}{ }^{2}$-, sollte Morelli kurz darauf im ersten Band seines Werkes Kunstkritische Studien über italienische Malerei ausführlich erörtern. ${ }^{23}$ Für Carlo Ginzburg wurde sie zum Indiz für ein Indizienparadigma Ende des 19. Jahrhunderts. ${ }^{24}$

\section{Anschauungsunterricht}

Jenseits aller Rhetorik bestehen wichtige Berührungen zwischen Universität und Schule bzw. Wissenschaft und Volksbildung, speziell Kunstgeschichte und Pädagogik. Sie begegnen sich insbesondere in ihrem Fokus auf das Bild und dem daraus resultierenden Rekurs auf das vergleichende Sehen. Gelehrte wie Anton

4 Heinrich Wölfflin, Kunstgeschichtliche Grundbegriffe, 1915. 
Springer oder Ferdinand Piper fordern bereits Mitte des 19. Jahrhunderts dazu auf, an den Schulen auch Kunstwerke zu berücksichtigen, z.T. auch Kunstgeschichte als Fach einzurichten, während Pädagogik und Schulbildung zunehmend explizit Bilderfragen in den Mittelpunkt ihrer Interessen rücken. ${ }^{25}$ Möglichkeiten und Grenzen eines 'Anschauungsunterrichts (auch und vor allem in Abgrenzung zu einem sanschaulichen Unterrichts), die Bedeutung von Anschauungsmaterial und Anschauungsmittel, Sehen lernen und Sehen lehren, Schulung der Augen etc. gehören zu den wiederkehrenden Topoi in diesem Zusammenhang. Explizit hat u.a. August Schmarsow die »Forderung eines kunsthistorischen Anschauungsunterrichts und geeigneter Seminarübungen im Sinne der ästhetischen Erziehung ${ }^{26}$ erhoben und seinen Seminarraum in Leipzig folgerichtig mit zahlreichen Bildflächen ausgestattet, »um möglichst viel Anschauungsmaterial gleichzeitig, für den Gang der Vorlesung oder Seminarübung geordnet, ausstellen zu können «: $:^{27}$

»Das erste Gebot dieser Anleitung zum Sehen der Kunstwerke bleibt aber, daß die sinnliche Erscheinung zu ihrem vollen Recht komme, und Anfang und Ende aller Betrachtung sei, d.h. daß das Bild, so inhaltreich es sein mag, nicht als Zeichen nur des Gedankengehaltes gefaßt werde, sondern um seiner selbst willen, und daß es zu Herz und Sinnen zugleich rede, wie ein Ereignis des Lebens in seiner vollen realen Wirklichkeit. Für den anders gewöhnten Betrachter giebt es allerdings kein besseres Mittel die Kunst des Sehens auszubilden, das Auge für das Bedeutsame in den Erscheinungen zu schärfen, als die Verbindung der Anschauung mit mündlicher Erklärung. $\aleph^{28}$

Projekte wie Seemanns Kunsthistorische Bilderbogen oder der genannte Kunstwart sind gleichermaßen Spiegel und Vehikel dieser Korrelation. Auch das spätere Engagement Alfred Lichtwarks im Kontext der Kunsterziehungsbewegung ist diesbezüglich symptomatisch. Eine frühe Mittlerfigur ist dagegen Bruno Meyer, der Herbartianer unter den frühen Kunsthistorikern, dem nicht zuletzt das Verdienst zukommt, die Diaprojektion in die (deutschsprachige) Kunstgeschichte eingeführt zu haben. ${ }^{29}$ Wiederholt wird Meyer im Kontext der Volksbildung als »autorität auf dem gebiet der kunstlehre und der ästhetischen pädagogik $\aleph^{30}$ gewürdigt. Viel besprochen wurde insbesondere sein Vortrag im Rahmen der 36. Philologenversammlung 1882 zum Thema Die Kunstwissenschaft und 
die Mittelschule, in dem Meyer-aus der Perspektive vom »ehemaligem praktischen Schulmanne» argumentierend-die Möglichkeiten, mittels Bildprojektion, "wissenschaftlich gearbeitetes und leidlich ausreichendes Bildermaterial« bereit zu stellen, ad oculos darlegen sollte. ${ }^{31}$ Das Vorhaben hatte er bereits anlässlich des ersten kunstwissenschaftlichen Kongresses in Wien 1873 vorgestellt, wie er später im Rahmen der Veröffentlichung seiner Glasphotogramme für den kunstwissenschaftlichen Unterricht im Projectionsapparat zu gebrauchen festhält: »Ich benutzte diese Gelegenheit, um, wie ich hiermit constatiren will, als der Erste in Deutschland, darauf hinzuweisen, welche grosse Wichtigkeit gerade für den kunstgeschichtlichen Unterricht der Projectionsapparat habe. ${ }^{32}$ Ungeachtet der in jüngster Zeit entfachten Prioritätenstreite um die Begründung der Dia-Doppelprojektion, ${ }^{33}$ wird man festhalten müssen, dass Meyer bereits die Einzelprojektion zu Vergleichszwecken nutzte, indem er sich montierter Gegenüberstellungen bediente. So setzte er unter anderem den zum damaligen Zeitpunkt höchst aktuellen und populären Vergleich von Dresdener und Darmstädter Holbein-Madonna gleich zweimal ins Bild. ${ }^{34}$

Es ist jedoch frappant, wie vehement im Zusammenhang mit Fragen des vergleichenden Sehens auf die eigene oder fremde Gründungsrolle verwiesen wird (Brethfeld, Woermann, Meyer etc.). ${ }^{35}$ Die Tendenz scheint sich heute auf einer Ebene zweiter Beobachtung fortzusetzen, wenn im Rahmen wissenschaftshistorischer Retrospektiven das vergleichende Sehen medientechnisch determiniert wird. So erklärte Heinrich Dilly mit Blick auf den Holbein-Streit: "Dass bei solchem vergleichenden Sehen die manuellen Reproduktionen der beiden Madonnen-Gemälde eher hinderlich, die fotografischen dagegen recht hilfreich waren, zählt zur faszinierenden Geschichte des Instrumentariums bzw. zur Geschichte kunsthistorischer Medien. $\aleph^{36}$ Die Fokussierung auf die neuen Medien ist jedoch bereits mit Blick auf das historische Bildmaterial bedenklich [Abb. 5]. Wie nicht zuletzt die visuellen Argumentationen und Reproduktionen im Rahmen des Holbein-Streits zeigen, begleiten vielschichtige Bilder-Kaskaden und vielfältige mixed-media-Formationen die kunsthistorische Forschung, so dass eine Polarisierung in manuelle und technische Verfahren ebenso problematisch erscheint wie die strikte Trennung künstlerischer und wissenschaftlicher Reproduktion. ${ }^{37}$ Nicht nur aus historischer Sicht, sondern auch methodologisch erscheint es kontraproduktiv, das vergleichende Sehen an einzelne Bildmedien zu knüpfen. Auch mit Blick auf das 

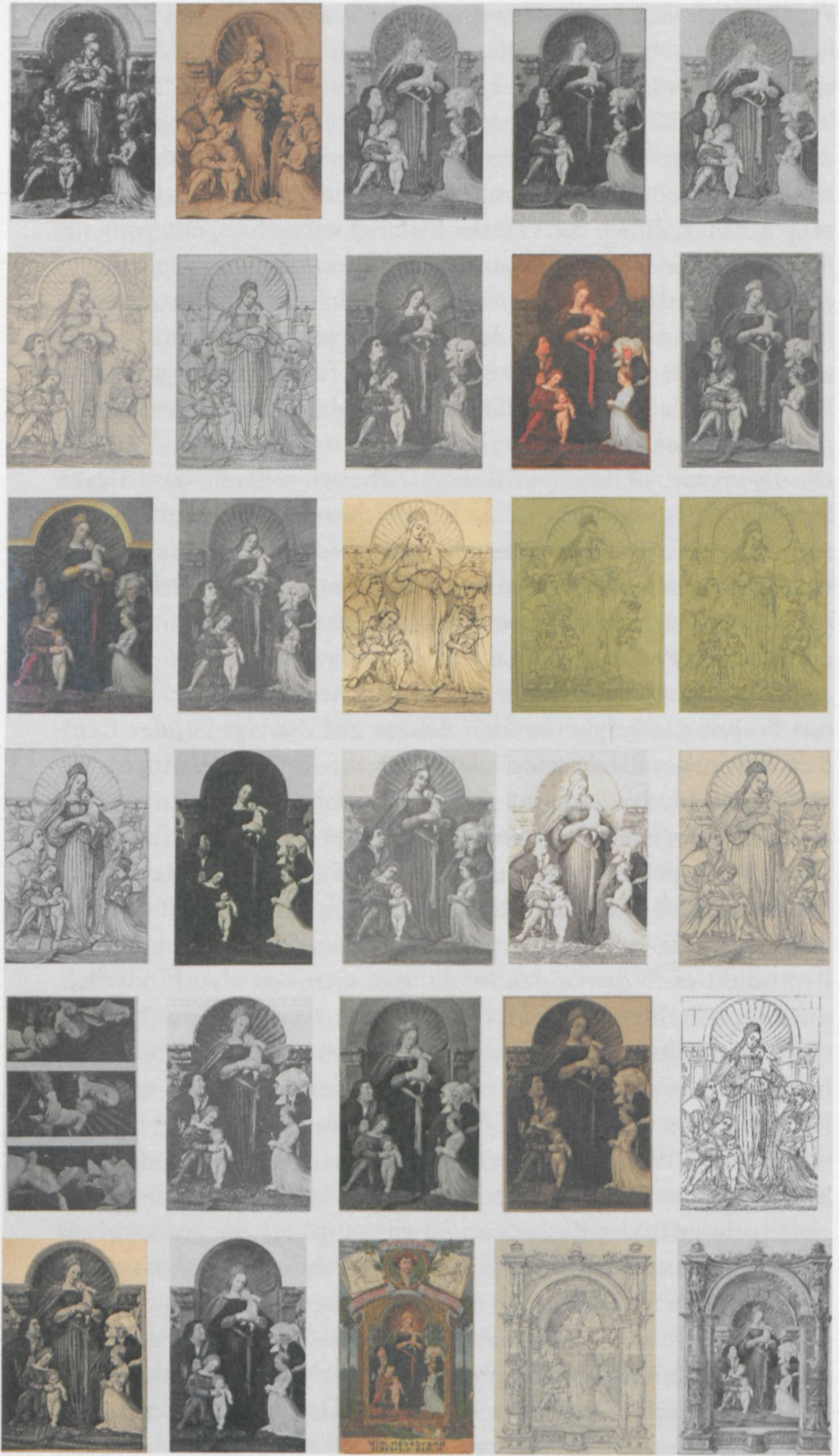

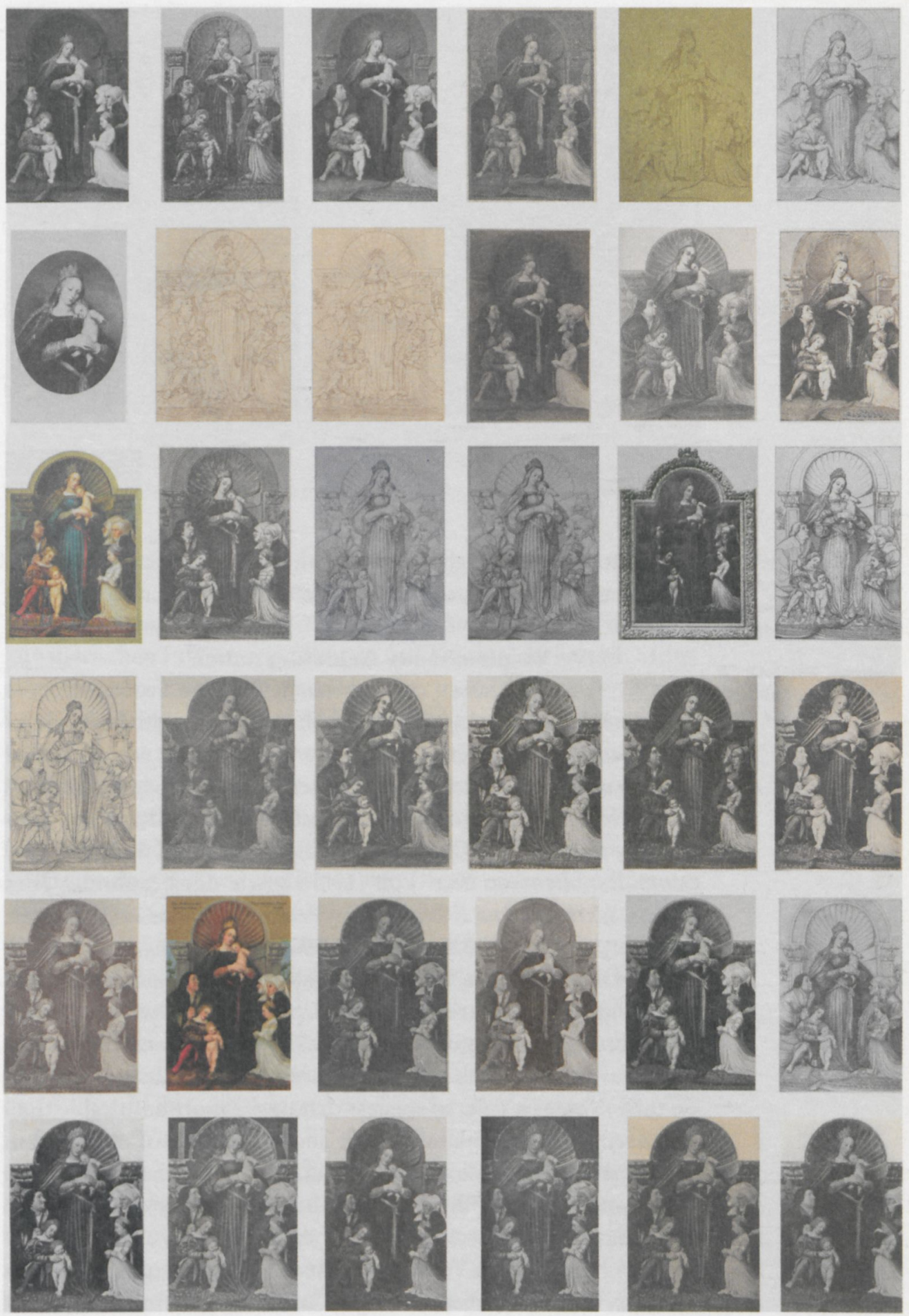
19. Jahrhundert sind neben der Einführung der neuen Medien Fotografie und Lichtbildprojektion weitere Motivationen zu befragen.

\section{Vergleichende Gemäldestudien}

Um 1900 lassen sich zahlreiche Projekte beobachten, die aus dem Dialog zwischen (akademischer) Kunstgeschichte und (allgemeiner) Pädagogik entstehen. In wenigen Jahren erscheint eine Fülle an reich bebilderten Experimenten im vergleichenden Sehen. ${ }^{38}$ Vor diesem Hintergrund entstehen auch die Publikationen How to study pictures von Charles H. Caffin (1908) und Vergleichende Gemäldestudien von Karl Voll (1907) sowie das berühmte Überblickswerk Sehen und Erkennen von Paul Brandt, das 1911 erstmals erscheint und bis 1968 in über 150.000 Exemplaren veröffentlicht wird.$^{39}$ Die von Caffin im Titel aufgeworfene Frage findet im vergleichenden Sehen ihre Antwort: "I propose that we shall study them through a series of comparisons. $\aleph^{40}$ Präzisierend fügt er hinzu: "I have adopted the parallel method: ,Look on this picture and on this. Not, as a rule, to suggest that one is more admirable than another; but to stimulate interest and the faculty of observation, and to show how various are the motives which have prompted artists and the methods which they have adopted $«{ }^{41}$ Caffins Buch war ebenfalls ein Bestseller, bis 1941 erscheint es in elf Auflagen. ${ }^{42}$

Wie Voll im Vorwort zu seinen eigenen Vergleichsstudien bemerkt, laufen die Untersuchungen Caffins darauf hinaus, »ethische 


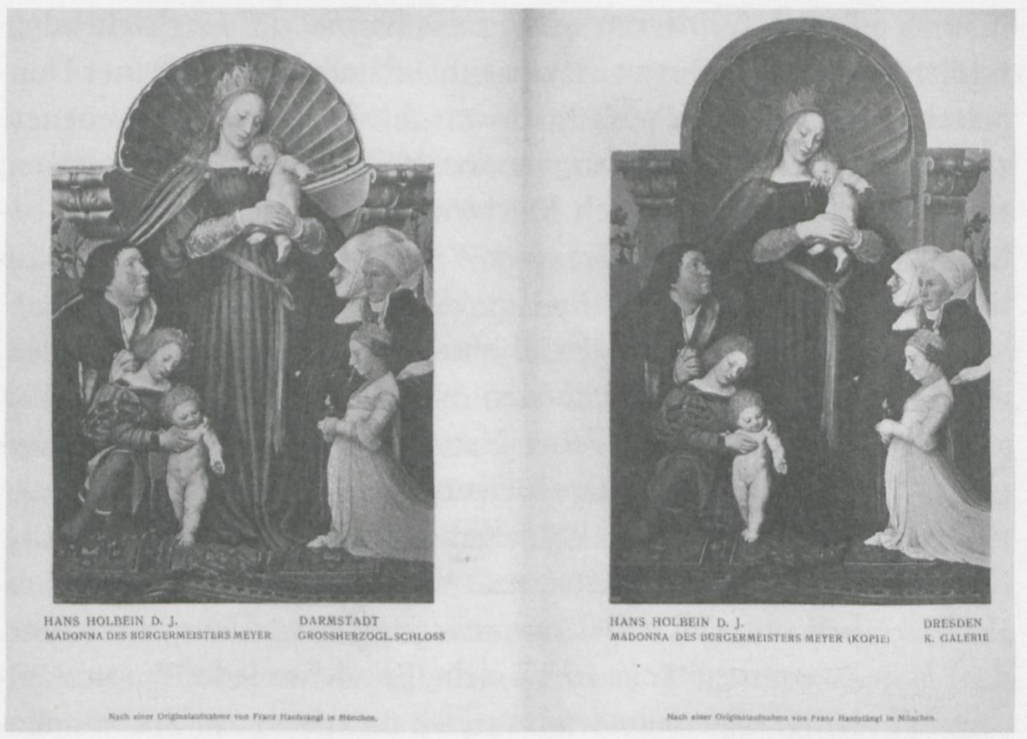

und kulturhistorische Betrachtungen vor den Bildern anzustellen « ${ }^{43}$ Davon grenzt sich der Münchener Kunsthistoriker erklärtermaßen ab, um-in Anlehnung an Wölfflin-sein Augenmerk auf die »rein künstlerischen Faktoren « zu richten, d.h. Kunst nicht »als ein Mittel zum Zweck, z. B. des Studiums der Kulturgeschichte « zu betrachten. Wie zuvor Schmarsow, spricht Voll sich zugunsten eines kunsthistorischen »Anschauungsunterrichtes « aus: »Die Versuchung liegt ja gerade in der Schule sehr nahe, das Kunstwerk als Illustration des vorgetragenen historischen Stoffes zu verwenden. ${ }^{44}$ Wie Caffin arbeitet auch Voll mit anschaulichen Gegenüberstellungen, die Bildpaare aber stellt er explizit in den Dienst einer "Schulung des Auges«: "Das Schwergewicht liegt eben doch auf den Abbildungen, die so herausgesucht wurden, daß das einfache Gegenüberstellen schon genügend Aufklärung bieten muß. Es handelt sich weniger um ein Buch zum Lesen als um eine Gelegenheit, das Auge zu üben. ${ }^{45}$ Als ein "Stück angewandter Kunstgeschichte" ergebe die Sammlung »bei richtigem Gebrauch der Tafeln [...] einen Leitfaden der Stilgeschichte der letzten Jahrhunderte «. ${ }^{46}$ Wenn auch Voll in der Folge Wölfflins »die Gleichberechtigung aller Stile« betont, grenzt er sich nicht im gleichen Maße gegen Werturteile ab. Die Darstellung zeigt sich belehrend, stellenweise gar autoritär, zugunsten einer Orientierung im "Widerstreit der Meinungen über das, was in der Kunst gut oder auch schön sei«. ${ }^{47}$ Das vergleichende Sehen wird dabei zum (vermeintlichen) Garant visueller Evidenz.

5 Vorherige Seite: Reproduktionen der Holbein-Madonna, 19. Jahrhundert.

6 Karl Voll, Vergleichende Gemäldestudien, 1907. 
Brandt wiederum sprengt das Format der vergleichenden Bildpaare und kombiniert bis zu acht Darstellungen auf einer Doppelseite, um Bilder nach Maßgabe verschiedener Vergleichsebenen zu kombinieren (nach Gattungen, nach Bildformen und Rahmen, regional und national, nach Motiven und Themen). Auch er betont explizit Erkenntniswert und Wirkungsmächtigkeit anschaulicher Gegenüberstellungen:

"Der Vergleich sagt viel ohne Worte, er macht auch den Stummen beredt, mögen die Vergleichspunkte formeller oder gegenständlicher Natur sein, mögen die verglichenen Kunstwerke eine fortlaufende Entwicklungsreihe oder entgegengesetzte Pole bilden, mögen sie gleichen oder verschiedenen Zeiten und Völkern entstammen. Und es braucht sich dabei keineswegs jedes Mal um historische Zusammenhänge zu handeln; gerade wo jeder Zusammenhang ausgeschlossen ist, treten die der Kunst innewohnenden Kräfte um so klarer hervor. « ${ }^{48}$

Es ist bezeichnend, dass Voll als erstes Beispiel seiner Vergleichende [n] Gemäldestudien das Paar der Holbein-Madonna wählt [Abb.6]. Auch Brandt verzichtet nicht darauf, wählt dazu aber die (ebenfalls paradigmatische) Gegenüberstellung von HolbeinMadonna und Sixtina. Die Holbein-Ausstellung in Dresden, die 1871 erstmals eine Gegenüberstellung der zwei Gemälde (im Original) ermöglicht hatte, war in vielerlei Hinsicht ein entscheidendes Vorbild. Die Ausstellung, die einen zweitägigen Holbein-Kongress veranlasste, wurde Auftakt zum kunsthistorischen Kongress, auf dem Meyer das Verfahren der Diaprojektion vorstellen sollte. Albert von Zahn, einer der zentralen Initiatoren, resümierte in seinem Ausstellungsbericht: »Vielleicht noch niemals hat in gleicher Weise wie bei der Holbein-Ausstellung sich der Vortheil gemeinsamer Prüfung, gemeinsamer wissenschaftlicher Beobachtung gezeigt. ${ }^{49}$ Als der »erste kunstwissenschaftliche Congress « zwei Jahre später in Wien tagte, berief man sich wiederholt auf das Dresdener Vorbild. ${ }^{50}$ Nicht nur die Zusammenkunft, sondern die Methode selbst wurde zum Vorbild:

»Wie Vieles auch die `Ergebnisse noch zu wünschen übrig lassen, diejenigen, welche sich in ernsthafter Weise an der gemeinsamen wissenschaftlichen Arbeit betheiligten, dürfen sich sagen, dass sie die Erkenntnis von dem Schaffen des grossen Meisters, dem die Ausstellung galt, ein 
gutes Stück gefördert haben und dass mit der gemeinsamen vergleichenden Forschung ein Weg betreten worden ist, auf welchem wir fernere Fortschritte in unserer Wissenschaft mit Zuversicht erhoffen dürfen. $\aleph^{51}$

Vielfach wurde im Kontext der späteren Forschung auf den »spektakulären Gemäldevergleich « ${ }^{52}$ verwiesen; inwiefern das vergleichende Sehen selbst zum Streitpunkt wurde, blieb dabei jedoch unberücksichtigt. ${ }^{53}$ Weitere Beispiele ließen sich nennen, um die Vielfalt der Variationen, Funktionen und Implikationen vergleichenden Sehens hervorzuheben und das Prinzip infolge der »doppelten Bedeutung des Vergleichs als Analyse- und Argumentationsmittel $\aleph^{54}$ im Sinne einer komplexen Sehtechnik zu würdigen. Die verbreitete Überzeugung, das "so oft beschworene Vergleichende Sehen ${ }^{55}$ gehöre zu den stillschweigenden, stillgeschwiegenen Techniken der Kunstgeschichte könnte vor diesem Hintergrund ebenso in Frage gestellt werden wie damit verbundene Vorurteile, denen man im Kontext der Forschung begegnet. ${ }^{56}$

\section{Kunstgeschichtliche Grundbegriffe}

$\mathrm{Zu}$ den bedeutendsten Etappen einer Bildgeschichte des vergleichenden Sehens gehören insbesondere Heinrich Wölfflins Kunstgeschichtliche Grundbegriffe von 1915 [Abb.4]. Am Beispiel vom »Stilwandel von der Renaissance zum Barock« erläutert Wölfflin-laut Martin Warnke »der unübertroffene Meister des vergleichenden Sehens ${ }{ }^{57}$-anhand anschaulicher Gegenüberstellungen "wie ein neuer Zeitgeist sich eine neue Form erzwingt $« .{ }^{58}$ Die Bild gewordenen "Prinzipien der vergleichenden Kunstgeschichte ${ }^{59}$ treten im Zusammenspiel von Bild und Text markant hervor. Die Wechselwirkung zwischen visueller Inszenierung und Beschreibung macht deutlich, wie sehr Wölfflin, wie Warnke schreibt, »immer wieder auf zwei Gegenbegriffe zusteuert: auf Fläche und Tiefe etwa; auf geschlossene Form und offene Form; auf Vielheit und Einheit; auf Klarheit und Unklarheit«. ${ }^{60}$

Man muss sich näher mit dem begleitenden Text auseinandersetzen, um Wölfflins Arbeit am »Kontrasteindruck « ${ }^{61}$ als intensive Durchdringung des vergleichenden Sehens zu erkennen. Seine Beschreibungen sind mehr als paarweise Schilderungen, sie sind wortgewandte und sprachkünstlerische Darstellungen einer Zusammenschau, die in der rhetorischen Komplizität als kunsthistorisch bedeutsames Ereignis hervortritt: 
»Um mit einem Vergleiche das Verhältnis klar zu machen: Das unfassbare Strömen der Kraft im Barock verhält sich zu der bestimmt gefassten Kraft der Renaissance wie die Lichtführung Rembrandts zur Lichtführung Lionardos: wo dieser in lauter klaren Formen modelliert, läßt jener das Licht in geheimnisvoll huschenden Massen über das Bild hingehen. Anders ausgedrückt: Klassische Klarheit heißt Darstellung in letzten bleibenden Formen, barocke Unklarheit heißt, die Form als etwas sich Veränderndes, Werdendes erscheinen lassen. $\ll^{62}$

Wölfflin hat explizit für und über seine Bilder argumentiert. An deren Bedeutung lässt er keinen Zweifel: Sie seien »immerhin zahlreich genug, um auch für sich allein ein Interesse wecken und über die Andeutungen des Textes hinaus den Leser zu eigenen Betrachtungen anregen zu können ${ }^{63}$ Die Art und Weise, in der Wölfflin den Dialog von Bild und Text arrangiert, ist bemerkenswert: Keine Abbildungsnummern oder in Klammern gesetzten Verweise, auch keine Angaben zu Herkunft oder Format und Medien finden (in der Regel) Eingang in den Haupttext-ausführlichere Informationen werden in das Abbildungsverzeichnis verlagert. Im Text werden die Bilder lediglich mit Nachname des Künstlers (»Tintoretto", "Bernini«) oder im Falle von Architekturen mit Ort und Name (»Rom, Fontana Trevi«) versehen. Bis auf wenige Ausnahmen wird (ab der zweiten, um einige Bilder bereicherten, Ausgabe von 1917) mit einem Sternchen auf den Bezug aufmerksam gemacht. Ohne Wölfflins aktive Mitgestaltung am Layout würde das ungezwungene Verweissystem nicht funktionieren. Er musste Wert darauf legen, dass die Bilder in unmittelbarer Nachbarschaft zu ihrer Besprechung auftauchen und dass dort, wo die Nähe nicht gewährleistet wurde, entsprechende Anmerkungen hinzugefügt wurden. ${ }^{64}$

Die spielerische Kombination der Bilder-auch Vergleiche, die »wie eine Blasphemie vorkommen ${ }^{65}$ können, werden eingebracht-ist Motor und Vehikel für das Spiel der Formen-eine Formel, auf die Wölfflin wiederholt zurückgreift, um die vergleichende Bilderfahrung in bemerkenswerten Bildbeschreibungen zu konzeptualisieren:

»die Form fängt an zu spielen, Lichter und Schatten werden zu einem selbständigen Element, sie suchen sich und binden sich, von Höhe zu Höhe, von Tiefe zu Tiefe; das Ganze gewinnt den Schein einer rastlos quellenden, nie 
endenden Bewegung. Ob die Bewegung flackernd und heftig sei oder nur ein leises Zittern und Flimmern: sie bleibt für die Anschauung ein Unerschöpfliches. ${ }^{66}$

Heinrich Alfred Schmid, Wölfflins Nachfolger in Basel, hat diesen Impuls wiederholt unterstrichen und als bedeutende Korrektur zu Morellis Fokussierung auf die Form gewürdigt-»also auch die Bewegung, nicht allein die Form $«{ }^{67}$ In dieser Dynamik, die Wölfflin unaufhörlich pointiert, artikuliert sich eine der wohl treffendsten Manifestationen des vergleichenden Sehens: "es ist eine Kraft in der Form, das Sehen aufzuwecken und zu einer einheitlichen Auffassung des Vielen zu zwingen, der sich auch ein stumpfer Betrachter kaum entziehen kann. Er wird munter und fühlt sich plötzlich als ein anderer Kerl. ${ }^{68}$ Die Dynamik, die das vergleichende Sehen im Dialog der Bilder entfalten kann-aus einer unaufhaltsamen Eigendynamik der Form begründet und auf diese Art in Wort und Bild zum Ausdruck gebracht-, macht den besonderen Reiz der Kunstgeschichtliche[n] Grundbegriffe aus.

\section{Visuelle Assoziationen}

Es ist insofern bezeichnend, dass Wölfflin später wiederholt gegen die durch das gedruckte Format erzwungene Stillstellung des Vergleichs rebellieren sollte. Aus einer dem vergleichenden Sehen inhärenten Dynamik begründet Wölfflin, 16 Jahre nach Erstveröffentlichung seiner Grundbegriffe, »die Scheu, im starren Zusammenhang eines Buches von diesem Mittel der Verdeutlichung einen allzu ausgedehnten Gebrauch zu machen. Schließlich ist es nicht nur eine Vergewaltigung des Lesers, sondern auch des Kunstwerks, wenn jedem Bild eine bestimmte Kontrastwirkung abgepresst wird «. ${ }^{69}$

Der Gedanke findet sich in Bruno Latours Theorie der »immutable mobiles « als grundlegendes Denkmodell wieder: »You have to invent objects, which have the properties of being mobile but also immutable, presentable, readable and combinable with one another. ${ }^{70}$ Analog dazu, aber mit einem stärkeren Fokus auf Fragen der Bilder, hat Horst Bredekamp am Beispiel der Kunstkammer die Idee von einem "Netz des assoziativen und grenzüberschreitenden visuellen Austausches « entfaltet. ${ }^{71}$ Einher mit dem Motiv vom »Denken in visuellen Assoziationen ${ }^{72}$-Latour spricht von Kaskadierungseffekten und der "connective quality « $^{73}$ - geht dabei die These vom "Sehen, Assoziieren und Denken als Spiel «. ${ }^{74}$ Die Beobachtung ist fundamental für das vergleichende Sehen. ${ }^{75}$ 
Wie Jacques Rancière darlegt, hat Jean-Luc Godard diese assoziative Kraft als »fraternité des métaphores ${ }^{76}$ prägnant auf den Punkt gebracht:

"la possibilité pour une attitude dessinée par le crayon de Goya de s'associer avec le dessin d'un plan cinématographique ou avec la forme d'un corps supplicié dans les camps nazis saisi par l'objectif photographique; la possibilité d'écrire de multiples façons l'histoire du siècle en vertu du double pouvoir de chaque image: celui de condenser une multiplicité de gestes significatifs d'un temps et celui de s'associer avec toutes les images douées du même pouvoir.«"

Wie nicht zuletzt Wölfflins spätere Anmerkung nahe legt, begründet das vergleichende Sehen aus dem Kontrast von Stillstellung und Oszillation, Antwort und Frage seine spezifische Wirkungsmächtigkeit, die somit in der Dia-Doppelprojektion, prägnanter als im gedruckten Format, als produktive Spannung erfahrbar wird. In Abgrenzung zu Brethfeld, der mit seinem zitierten Klassifizierungsversuch das vergleichende Sehen gegen ein »bloßes Basteln" abzugrenzen versucht, ließe sich hier mit Claude LéviStrauss das Prinzip der »bricolage« als »eine Art intellektueller Bastelei« fruchtbar machen, »um eine nicht vorgezeichnete Bewegung zu betonen $«{ }^{78}$ Für das vergleichende Sehen wäre demzufolge mit 


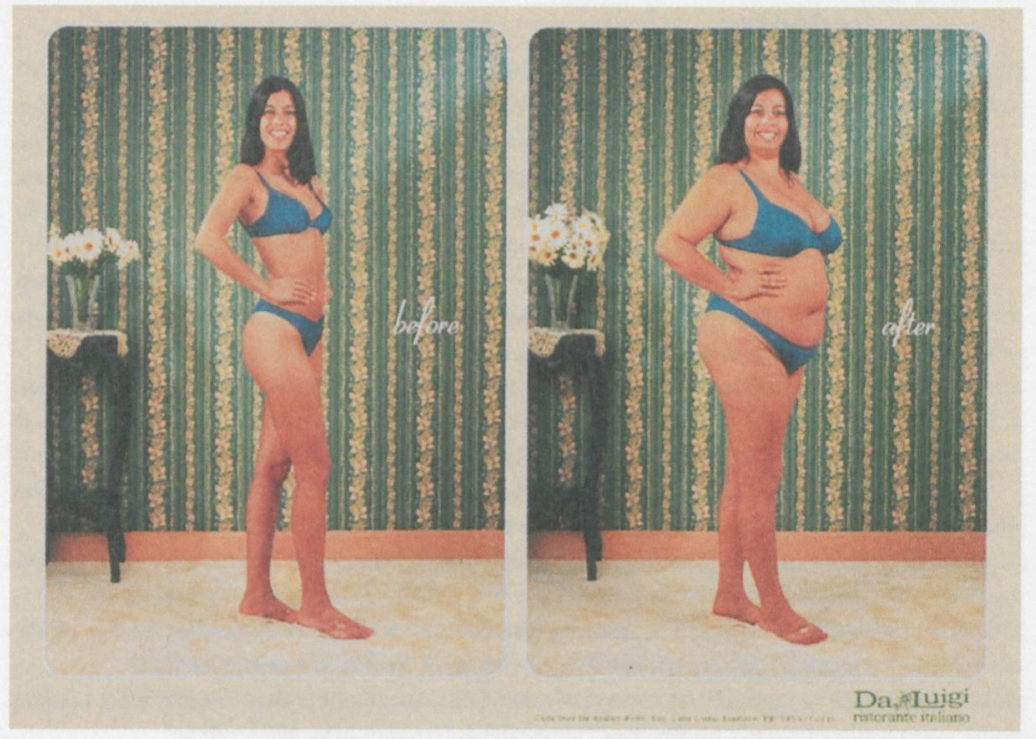

Nachdruck »das Recht zur Folgerung« zu reklamieren: »das Recht nämlich, zu postulieren, daß diese sichtbaren Merkmale auf besondere, doch verborgene Eigenschaften hinweisen $\aleph^{79}$ - man könnte mit Schmarsow hinzufügen: »solange das Auge allein in seinem Recht ist «. ${ }^{80}$

Spielerische Variationen anschaulicher Gegenüberstellungen, wie man sie insbesondere auch im Bereich der Unterhaltungsindustrie finden kann, erweisen sich vor diesem Hintergrund als Ausdruck einer wichtigen visuellen Erfahrung, durch die das vergleichende Sehen eigendynamisch und schöpferisch hindurchwirkt. Als abschließendes Beispiel sei dazu auf eine Restaurantwerbung der südamerikanischen Werbeagentur Badillo Nazca Saatchi \& Saatchi verwiesen [Abb. 7] ${ }^{81}$ Sie präsentiert sich-nicht zuletzt durch die Adaption einer Ikonografie stereoskopischer Fotografien des 19. Jahrhunderts - als humoristischer Kommentar auf Beweisführungen mittels anschaulicher Gegenüberstellungen, wie man sie insbesondere aus dem Bereich der Diätindustrie kennt, um diese in einem effektvollen détournement aufs Spiel zu setzen. Die assoziationsreiche Inszenierung ist Teil einer höchst aktuellen Tradition: Sie versteht das vergleichende Sehen als »intuitive Methode ${ }^{82}-$ nicht bloß als "rationelle Methode» (Schmid) - und weiß diese im Sinne einer bildschöpferischen »Variation über das Thema des Kausalitätsprinzips ${ }^{83}$ zugunsten einer spielerischen bricolage mit Bildern ernst zu nehmen. 


\section{Endnoten}

1 William J.T. Mitchell, Why Comparisons Are Odious, in: World Literature Today 70/2, 1996, S. 321-324, hier S.323. Siehe dazu Nelson Goodman, Seven strictures on similarity, in: ders., Problems and Projects, New York 1972, S. 437-446. Zu den Bedenken gegenüber komparatistischen Zugängen aus kunsthistorischer Perspektive zuletzt Marc Bayard, Les enjeux du comparatisme en histoire de l'art, in: ders. (Hg.), L'histoire de l'art et le comparatisme. Les horizons du détour, Rom/Paris 2007, S.9-21.

2 Helga Lutz, Jan-Friedrich Missfelder und Tilo Renz, Einleitung: illegitimes Vergleichen in den Kulturwissenschaften, in: dies. (Hg.), Illegitimes Vergleichen in den Kulturwissenschaften, Bielefeld 2006, S.7-20, hier S.9.

3 Katharina Krause, Argument oder Beleg. Das Bild im Text der Kunstgeschichte, in: dies., Klaus Niehr und Eva-Maria Hanebutt-Benz (Hg.), Bilderlust und Lesefrüchte. Das illustrierte Kunstbuch von 1750 bis 1920, Leipzig 2005, S. 27-43, hier S. 33.

4 Siehe hierzu den Beitrag von Peter Geimer in diesem Band sowie Matthias Bruhn, Das Bild: Theorie-Geschichte-Praxis, Berlin 2009, S. 146, 156.

5 André Malraux, Das imaginäre Museum (Psychologie der Kunst, Bd. 1) [1947], Hamburg 1957, S.22.

6 Robert S. Nelson, The Slide Lecture, or The Work of Art History in the Age of Mechanical Reproduction, in: Critical Inquiry 3, 2000, S.414-434, hier S. 422.

7 Claus Leggewie und Elke Mühlleitner, Die akademische Hintertreppe. Kleines Lexikon des wissenschaftlichen Kommunizierens, Frankfurt a.M. 2007, S.70f.

8 Felix Thürlemann, Bild gegen Bild, in: Aleida Assman, Ulrich Gaier und Gisela Trommsdorff (Hg.), Zwischen Literatur und Anthropologie. Diskurse, Medien, Performanzen, Tübingen 2005, S. 163-174, hier S.167. In diesem Sinne auch Malraux: „Bilder einander gegenüberzustellen ist ein intellektueller Prozeß und steht als solcher in grundsätzlichem Gegensatz zu jener Hingabe, aus der allein Versenkung möglich wird«. Malraux, Das imaginäre Museum (Anm. 5), S.9.

9 Andreas Beyer, Die sichtbaren Städte: Architekturgeschichte als Bildwissenschaft, in: Oskar Bätschmann (Hg.), Dienstleistung Kunstgeschichte? Art History on Demand?, Emsdetten 2008, S.99-105, hier S. 99 (in Bezug auf die in Italo Calvinos Roman evozierten Bildvergleiche).

10 Horst Bredekamp, Birgit Schneider und Vera Dünkel (Hg.), Das Technische Bild. Kompendium zu einer Stilgeschichte wissenschaftlicher Bilder, Berlin 2008, S. 26; bes. S. 2428 „Vergleich als Methode".

11 Krause, Argument oder Beleg (Anm. 3), bes. S. 32f. „Der Vergleich-ein Ausweg «.

12 Georges Didi-Huberman, Vor einem Bild, Wien 2000, S.28. Siehe hierzu die Differenzierung von sichtbarer Information (visible) und phänomenologischem Ereignis (visuel): ders., Bilder trotz allem, München 2007, S. 100-131.

13 Ebd., S.8f.

14 Lena Bader, Hat die Wissenschaftsgeschichte der Kunstgeschichte keine Lust am Bild?, in: Kunstgeschichte. Texte zur Diskussion, 2009-17 (urn:nbn:de:0009-23-17713), unter: http://www.kunstgeschichte-ejournal.net/discussion/2009/bader [05.05.2009].

15 Max Brethfeld, Vergleichendes Sehen im Dienste der Augen-, Geistes- und Geschmacksbildung, in: Die Arbeitsschule 3, 1931, S.124-128, hier S. 125.

16 Die starke Emphase auf Führertum und nationale Volksbildung wird umso deutlicher in: Max Brethfeld, Anschauungs- und Uebungsmittel fuer eine volkstuemliche Formen- und Raumkunde, Leipzig 1934. Für eine Diffamierung missbraucht das vergleichende Sehen insbesondere Paul Schultze-Naumburg, Kunst und Rasse, München 1928.

17 Brethfeld, Vergleichendes Sehen (Anm.15) 1931, S.124f.

18 Ebd., S. $126 f$.

19 Ebd., S. 128.

20 Karl Woermann, Selbstdarstellung, in: Johannes Jahn (Hg.), Die Kunstwissenschaft der Gegenwart in Selbstdarstellungen, Leipzig 1924, S.198-227, hier S.210f. Woermann verweist in diesem Zusammenhang zudem auf Heinrich Brunn, von dem er-gemeinsam 
mit Adolf Furtwängler-»erst methodisch sehen und vergleichen " gelernt habe (S.203). Explizit würdigt er dabei auch die Rolle von "Photographien als Vergleichsmaterial« und damit einhergehenden $»$ Vergleichsreisen $*(S .212,218)$.

21 Karl Woermann, Katalog der Königlichen Gemäldegalerie zu Dresden, Dresden 1887, S.XII.

22 Heinrich Alfred Schmid, Kunstsammlungen, Kunstwissenschaft und Kunstunterricht, Basel 1935, S.23.

23 Giovanni Morelli, Kunstkritische Studien über italienische Malerei, Bd. 1: Die Galerien Borghese und Doria Panfili in Rom, Leipzig 1890, bes. S. 1-78 "Princip und Methode«.

24 Carlo Ginzburg, Spurensicherung. Der Jäger entziffert die Fährte, Sherlock Holmes nimmt die Lupe, Freud liest Morelli-die Wissenschaft auf der Suche nach sich selbst, in: ders., Spurensicherungen. Über verborgene Geschichte, Kunst und soziales Gedächtnis, München 1988, S. 78-126.

$25 \mathrm{Zu}$ den paradigmatischen Positionen dieser Diskussion gehören u.a. Bernhard Stark, Kunst und Schule: Zur deutschen Schulreform, Jena 1848; Anton Springer, Der Kunstunterricht auf gelehrten Schulen, in: Recensionen und Mittheilungen über bildende Kunst 3, 1864, S. 153-155, 169-172; Bruno Meyer, Aus der ästhetischen Pädagogik, Berlin 1873. Siehe hierzu u.a. Rudolf Menge, Kunstunterricht am Gymnasium, in: Wilhelm Rein (Hg.), Encyklopädisches Handbuch der Pädagogik, Bd. 5, Langensalza 1906, S. 250-265; mit einer Übersicht über einzelne Beiträge Franz Müller, Uber den sogenannten Kunstunterricht auf Gymnasien, in: Hermann Masius (Hg.), Jahrbücher für Philologie und Paedagogik. Zweite Abtheilung, Leipzig 1883, S.416-425, 472-480, 511-517.

26 August Schmarsow, Die Kunstgeschichte an unsern Hochschulen, Berlin 1891, S. 117.

27 August Schmarsow, Das kunsthistorische Institut, in: Festschrift zur Feier des 500jährigen Bestehens der Universität Leipzig, hg.v. Rektor und Senat, Bd.4, Teil 1, Leipzig 1909, S. 176. Vgl.Abb. 5 im Beitrag von Klaus Niehr in diesem Band.

28 Schmarsow, Die Kunstgeschichte an unsern Hochschulen (Anm. 26), S. 115.

29 Siehe hierzu Meyer, Aus der ästhetischen Pädagogik (Anm.25). Eine frühe Untersuchung dieser Beziehung bietet Marie Luise Eichelbaum, Die Lehre von der ästhetischen Erziehung bei den Herbartianern und bei Bruno Meyer, Bonn 1922. Zu berücksichtigen wären in diesem Zusammenhang auch zeitgleiche Diskussionen um Déjà-vu-Phänomene und Geisterfotografie. Einführend dazu Günther Oesterle und Lothar Schneider, Einleitung, in: ders. (Hg.), Déjà-vu in Literatur und bildender Kunst, München 2003, S.7-27; Thomas Trummer, Déjà-vu. Menschliche Erinnerung und apparative Speicherungstechnik, in: Déjà-vu. Der Augenblick der Nachträglichkeit in der zeitgenössischen Kunst, Wien 2005, S.6-15 [Katalog zur gleichnamigen Ausstellung, Atelier Augarten. Zentrum für zeitgenössische Kunst der Österreichischen Galerie Belvedere, 2005/06].

30 Müller, Über den sogenannten Kunstunterricht (Anm. 25), S. 479.

31 Bruno Meyer, Die Kunstwissenschaft und die Mittelschule, Vortrag gehalten am 29. September 1882, in: Verein Deutscher Philologen und Schulmänner, Verhandlungen der 36. Versammlung Deutscher Philologen und Schulmaenner, Leipzig 1883, S. 204-206, hier S. $204 f$.

32 Bruno Meyer, Glasphotogramme für den kunstwissenschaftlichen Unterricht, im Projectionsapparat zu gebrauchen, Karlsruhe 1883, S.IV. Siehe dazu Erster kunstwissenschaftlicher Congress in Wien, in: Mittheilungen des K. K. Österreichischen Museums für Kunst und Industrie 5, 1874/1875, S.9-22, bes. S.9-13; Bruno Meyer, Die Photographie im Dienste der Kunstwissenschaft und des Kunstunterrichtes, in: Westermann's illustrierte deutsche Monatshefte 47, 1879/1880, S. 196-209, 307-318, bes. S.310. Siehe dazu Martin Papenbrock, Der Lehrstuhl für Kunstgeschichte in Karlsruhe-ein Rückblick, in: ders. und Katharina Büttner (Hg.), Kunst und Architektur in Karlsruhe. Festschrift für Norbert Schneider, Karlsruhe 2006, S. 181-193.

33 Siehe dazu Heinrich Dilly, Weder Grimm, noch Schmarsow, geschweige denn Wölfflin... Zur jüngsten Diskussion über die Diaprojektion um 1900, in: Costanza Caraffa 


\section{Endnoten}

(Hg.), Fotografie als Instrument und Medium der Kunstgeschichte, München/Berlin, 2009, S.91-116.

34 Siehe hierzu im Katalog die Angaben zu den Nummern 3809 und 3810: Meyer, Glasphotogramme (Anm. 32), S. 122.

35 Richard Hamann und Jost Hermand wiederum betonten, Julius Meier-Graefe habe wzum ersten Mal die vergleichende Bildbetrachtung zum obersten Prinzip der kunstwissenschaftlichen Analyse erhoben «. Richard Hamann und Jost Hermand, Deutsche Kunst und Kultur von der Gründerzeit bis zum Expressionismus, Bd. 3: Impressionismus, Berlin 1960, S. 104.

36 Heinrich Dilly, Bildgeschichten und Bildkritik der traditionellen Kunstgeschichte, in: Matthias Bruhn (Hg.), Sichtbarkeit der Geschichte. Beiträge zu einer Historiografie der Bilder, Berlin 2005, S. 15-26, hier S. 18f. Noch expliziter an die neuen Medien knüpfen das vergleichende Sehen Wolfgang Ernst und Stefan Heidenreich, Digitale Bildarchivierung. Der Wölfflin-Kalkül, in: Sigrid Schade und Georg C. Tholen (Hg.), Konfigurationen: zwischen Kunst und Medien, München 1999, S. 306-320.

37 Siehe hierzu Lena Bader, Kopie und Reproduktion im Holbein-Streit. Eine wissenschaftshistorische Retrospektive aus bildkritischer Perspektive, in: Wojciech Balus und Joanna Wolanska (Hg.), Die Etablierung und Entwicklung des Faches Kunstgeschichte in Deutschland, Polen und Mitteleuropa, (erscheint) Krakau 2009.

38 Siehe hierzu eine bibliografische Auswahl am Ende dieses Bandes.

39 Siehe hierzu Magdalena Bushart, Die Oberfläche der Bilder. Paul Brandts vergleichende Kunstgeschichte, in: Kritische Berichte 37/1, 2009, S.36-54.

40 Charles Henry Caffin, How to study pictures. By means of a series of comparisons of paintings and painters from Cimabue to Monet, with historical and biographical summaries and appreciations of the painter's motive and methods, New York 1908, S. 6.

41 Ebd., S.XIV.

42 Siehe Astrit Schmidt-Burkhardt, Stammbäume der Kunst: Zur Genealogie der Avantgarde, Berlin 2005, S. 285.

43 Karl Voll, Vergleichende Gemäldestudien, München/Leipzig 1907, S. 15.

44 Ebd., S. $14 f$.

45 Voll, Vergleichende Gemäldestudien (Anm.43), S.11.

46 Ebd., S. 12.

47 Ebd., S. 18. Wie Voll explizit markiert, bedeutet seine Abgrenzung gegen ältere kulturhistorische Ansätze nicht eine Verschiebung zugunsten einer »rein formale[n] Behandlung«. Stattdessen sucht er gezielt eine Kombination der »vormals unberücksichtigten technischen Fragen « mit dem »geistigen Inhalt eines Kunstwerkes«. Ebd., S. 18f.

48 Paul Brandt, Sehen und Erkennen. Eine Anleitung zu vergleichender Kunstbetrachtung [1911], Leipzig 1913, S.Vf.

49 Albert von Zahn, Die Ergebnisse der Holbein-Ausstellung zu Dresden. II, in: Jahrbücher für Kunstwissenschaft 3, 1873, S. 193-215, hier S. 215.

50 Wie u. a. das Beiblatt zur Zeitschrift für bildende Kunst am 11.07.1873 berichtet, wurde der wimprovisierte[ ] Kunstforschertag in Dresden « zum Anlass, »derartige Versammlungen in festerer Gestalt und mit bestimmten Programm zu wiederholen «. Kunstwissenschaftlicher Congreß in Wien, in: Zeitschrift für bildende Kunst. Mit dem Beiblatt Kunst-Chronik 8, 1873, S.617.

51 Zahn, Die Ergebnisse der Holbein-Ausstellung (Anm.49), S.215.

52 Udo Kultermann, Geschichte der Kunstgeschichte. Der Weg einer Wissenschaft, München 1990, S. 136.

53 Siehe dazu Lena Bader, „die Form fängt an zu spielen ...« Kleines (wildes) Gedankenexperiment zum vergleichenden Sehen, in: Bildwelten des Wissens, hg.v. Horst Bredekamp, Matthias Bruhn und Gabriele Werner, Bd.7,1: Bildendes Sehen, Berlin 2009, S. 35-44.

54 Bredekamp, Schneider und Dünkel, Das Technische Bild (Anm. 10), S.24. Siehe hierzu eine bibliografische Auswahl am Ende dieses Bandes. 
55 Dilly, Weder Grimm, noch Schmarsow (Anm. 33), S. 107.

56 Exemplarisch dazu: »Ich betreibe also vvergleichendes Sehen - -eine legitime Methode der Kunstwissenschaft, die freilich zweifache Gefahren mit sich bringt: einerseits die Gefahr, in die Attitude des sdéjà vu zu fallen, in die blasierte Ablehnung der Gegenwart, weil alles doch schon dagewesen sei; auf der anderen Seite verleitet die Nähe zur Moderne womöglich zu voreiligen Schlüssen auf Sinn und Bedeutung des Alten, zu einem Snobismus der Naseweisheit etwa der Art, als hätten wir-sagen wir mit einer Skulptur von Constantin Brancusi-auch schon das Geheimnis der zykladischen Idole enthüllt «. Eduard Trier, Motivwanderungen aus der Antike ins 20. Jahrhundert, in: Peter Bloch (Hg.), Festschrift für Heinz Ladendorf, Köln/Wien 1970, S.129-136, hier S. 129f. Die Verfahren des vergleichenden Sehens als »blinde Flecken der kunstgeschichtlichen Praxis« markiert insbesondere Felix Thürlemann, Vom Einzelbild zum Hyperimage. Eine neue Herausforderung für die Kunstgeschichtliche Hermeneutik, in: Ada Neschke-Hentschke (Hg.), Les Herméneutiques au Seuil du XXIème Siècleévolution et débat actuel, Löwen/Paris 2004, S.223-248, hier S. 226.

57 Martin Warnke, Warburg und Wölfflin, in: Horst Bredekamp, Michael Diers und Charlotte Schoell-Glass (Hg.), Aby Warburg. Akten des internationalen Symposions Hamburg 1990, Weinheim 1991, S. 79-86, hier S. 83.

58 Heinrich Wölfflin, Kunstgeschichtliche Grundbegriffe. Das Problem der Stilentwicklung in der neueren Kunst [1915], Vierte Auflage, München 1920, S.9.

59 Heinrich Wölfflin, zit. nach Nikolaus Meier, Heinrich Wölfflin (1864-1945), in: Heinrich Dilly (Hg.), Altmeister der Kunstgeschichte, Berlin 1999, S.63-79, hier S. 70.

60 Warnke, Warburg und Wölfflin (Anm. 57), S. 83.

61 Wölfflin, Kunstgeschichtliche Grundbegriffe (Anm. 58), S. 147.

62 Ebd., S.238, in Bezug auf Bramante und den Dresdener Zwinger (ohne Abbildungen).

63 Ebd., S.VII.

64 Siehe u.a. die Ausnahmefälle ebd., S. 43 ("Abb. auf S. 1 "), S. 47 ("dessen Abbildung wir oben gebracht haben «), S. 73 ("Abb.S.241 u.S.242«), S. 158 ("Wir verweisen auf die Illustrationen auf Seite 64/65 zurück «).

65 Ebd., S. 188.

66 Ebd., S.21. Wie Wölfflin in Vorwort und Einleitung explizit hervorhebt, geht es ihm als »Formpsychologen" (S.3)-nicht Formhistoriker-nicht allein um die Entwicklung der Formenauffassung und die "Bestimmung individueller Stiltypen «, Volksstile oder Zeitstile. Vielmehr sollen mit den Formvorstellungen auch der »Formenrhythmus «, vor allem aber Bildvorstellungen selbst in den Mittelpunkt rücken: „die veränderte Bildgestaltung im allgemeinen, der Wechsel der Bildvorstellung überhaupt « (S. VII). Am Beispiel der Skulptur markiert er explizit die Verschiebung von der Figur zur Form, von einer Geschichte der objektiven Themen zur Stilentwicklung (S.113).

67 Schmid, Kunstsammlungen, Kunstwissenschaft und Kunstunterricht (Anm. 22), S. 25. Siehe dazu auch Heinrich Wölfflin, Grundbegriffe, in: ders., Gedanken zur Kunstgeschichte. Gedrucktes und Ungedrucktes, Basel 1941, S.7-24.

68 Wölfflin, Kunstgeschichtliche Grundbegriffe (Anm. 58), S. 167. In diesem Sinne erklärt Franz Roh, der in München als Wölfflins Assistent tätig war und bei diesem promovierte, es wäre »für die Erkenntnis reiner Formfragen [...] reizvoll, eine Kunstgeschichte der sich dauernd wandelnden Ausdruckweisen zu entwickeln [...], wobei die Abbildungen à la Leporello angeordnet sein müssten «. Franz Roh, Bildvergleiche, in: Die Kunst und das schöne Heim 48/4, 1950, S. 139-141, hier S. 139.

69 Heinrich Wölfflin, Die Kunst der Renaissance. Italien und das deutsche Formgefühl, München 1931, S.VI.

70 Bruno Latour, Drawing things together, in: Michael Lynch und Steve Woolgar ( $\mathrm{Hg}$.), Representation in scientific practice, Cambridge (Mass.) 1990, S. 19-68, hier S.26. Sein Fazit lautet konsequent: »If you want to understand what draws things together, then look what draws things together«. Ebd., S. 60. 


\section{Endnoten/Abbildungsnachweis}

71 Horst Bredekamp, Die Kunstkammer als Ort spielerischen Austauschs, in: Thomas W. Gaehtgens (Hg.), Künstlerischer Austausch. Artistic Exchange. Akten des XXVIII. Internationalen Kongresses für Kunstgeschichte. Berlin, 15.-20. Juli 1992, Bd. 1, Berlin 1993, S. 65-79, hier S. 66.

72 Ebd., S. 72.

73 Ebd., S. 54 .

74 Ebd., S.65.

75 In diesem Sinne mit Blick auf Morelli und Warburg ebd., S.71,

76 Jean-Luc Godard, Histoire(s) du cinéma, Bd.3: La monnaie de l’absolu, une vague nouvelle, Paris 1999, S. 149.

77 Jacques Rancière, L'image pensive, Vortrag gehalten am 08.10.2007 am NFS Bildkritik, Basel. Unveröffentlichtes Manuskript, S. [15].

78 Claude Lévi-Strauss, Das wilde Denken, Frankfurt a.M. 1973, S. 29.

79 Ebd., S. 28 .

80 Schmarsow, Die Kunstgeschichte an unsern Hochschulen (Anm. 26), S.61.

81 Badillo Nazca Saatchi \& Saatchi, before \& after, Da Luigi Ristorante, September 2003.

82 Lévi-Strauss, Das wilde Denken (Anm.78), S. 107.

83 Henri Hubert und Marcel Mauss in der Beschreibung des magischen Denkens, zit. ebd., S. 22.

\section{Abbildungsnachweis}

1 Burton Holmes um 1930, in: Burton Holmes, Reiseberichte: der größte Reisende seiner Zeit, 1892-1952, hg.v. Genoa Caldwell, Köln u. a. 2006, S. 13.

2 Richard Friebe, Zwei Bilder sagen mehr als tausend Worte, in: Frankfurter Allgemeine Zeitung, 13.06.2005, Abb.5, unter: http://www.faz.net/s/Rub2542FB5D98194DA3A1 F14B5B01EDB3FB/Doc E5EF601A2231947B7963DD06789C94C61 AT pl Eco mmon Sspezial.html [5.5.2009].

3 Giovanni Morelli, Kunstkritische Studien über italienische Malerei, Bd. 3: Die Galerien zu Berlin, hg.v. Gustavo Frizzoni, Leipzig 1893, S. 58.

4 Heinrich Wölfflin, Kunstgeschichtliche Grundbegriffe. Das Problem der Stilentwicklung in der neueren Kunst [1915], München 1920, S. 50-51.

5 Reproduktionen der Holbein-Madonna, 1723-1910 (Montage: Lena Bader).

6 Karl Voll, Vergleichende Gemäldestudien, München/Leipzig 1907, Abb. 1 und Abb.2, S. [208-209].

7 Badillo Nazca Saatchi \& Saatchi, before \& after, Da Luigi Ristorante, September 2003. Creators Copywriter: Christine Hasben, Art Director: Mariel Lebron, Creative Director: Juan Carlos Rodriguez, Typographer: Carlos Nieves, Illustrator: Carlos Nieves Advertising Agency. 